\title{
Scaling of the superconducting gap with orbital character in FeSe
}

\author{
Luke C. Rhodes, ${ }^{1,2}$ Matthew D. Watson,,${ }^{1,3, *}$ Amir A. Haghighirad, ${ }^{4,5}$ \\ Daniil V. Evtushinsky, ${ }^{6}$ Matthias Eschrig, ${ }^{2}$ and Timur K. Kim ${ }^{1, \dagger}$ \\ ${ }^{1}$ Diamond Light Source, Harwell Campus, Didcot OX11 ODE, United Kingdom \\ ${ }^{2}$ Department of Physics, Royal Holloway, University of London, Egham, Surrey TW20 0EX, United Kingdom \\ ${ }^{3}$ School of Physics and Astronomy, University of St. Andrews, St. Andrews KY16 9SS, United Kingdom \\ ${ }^{4}$ Clarendon Laboratory, Department of Physics, University of Oxford, Parks Road, Oxford OX1 3PU, United Kingdom \\ ${ }^{5}$ Institute for Solid State Physics, Karlsruhe Institute of Technology, D-76021 Karlsruhe, Germany \\ ${ }^{6}$ Institute of Physics, Ecole Polytechnique Federale Lausanne, CH-1015 Lausanne, Switzerland
}

(Received 3 April 2018; revised manuscript received 5 October 2018; published 14 November 2018)

\begin{abstract}
We use high-resolution angle-resolved photoemission spectroscopy to map the three-dimensional momentum dependence of the superconducting gap in FeSe. We find that on both the hole and electron Fermi surfaces, the magnitude of the gap follows the distribution of $d_{y z}$ orbital weight. Furthermore, we theoretically determine the momentum dependence of the superconducting gap by solving the linearized gap equation using a tightbinding model which quantitatively describes both the experimental band dispersions and orbital characters. By considering a Fermi surface only including one electron pocket, as observed spectroscopically, we obtain excellent agreement with the experimental gap structure. Our finding of a scaling between the superconducting gap and the $d_{y z}$ orbital weight supports the interpretation of superconductivity mediated by spin fluctuations in FeSe.
\end{abstract}

DOI: 10.1103/PhysRevB.98.180503

Over the last ten years of extensive studies on the ironbased superconductors, many experimental works have provided support for the spin-fluctuation pairing hypothesis, including the observation of a spin-resonance peak in the superconducting (SC) state [1,2], the presence of nodes in the SC gap in some systems [3], evidence for a sign change of the SC gap from quasiparticle interference [4], as well as the more general observation of SC in close proximity to antiferromagnetism in the phase diagrams [5]. A distinctive feature of spin-fluctuation mediated SC is that the pairing interactions are sensitive to the orbital character of the bands [6-8]. Fundamentally, this sensitivity arises since the relevant interaction is the strong, local, and instantaneous Coulomb repulsion, which will generally be larger for the pairing of electrons in the same orbital [9]. Thus an observation of a direct relationship between the orbital character and the SC gaps around the Fermi surface (FS) would constitute strong evidence for pairing by spin fluctuations in the Fe-based SC. In most Fe-based SC, the complexities of the multiband, multiorbital electronic structures makes the experimental verification of this crucial link challenging. However, here we focus on FeSe, which exhibits both nematic order and SC [10], and is known to have a relatively simple but highly anisotropic FS

\footnotetext{
*Corresponding author: mdw5 @st-andrews.ac.uk

${ }^{\dagger}$ Corresponding author: timur.kim@diamond.ac.uk
}

Published by the American Physical Society under the terms of the Creative Commons Attribution 4.0 International license. Further distribution of this work must maintain attribution to the author(s) and the published article's title, journal citation, and DOI.
[11-14], allowing detailed testing of the relationship between orbital character and SC [15-17].

In this Rapid Communication we present high-resolution angle-resolved photoemission spectroscopy (ARPES) measurements of the momentum dependence of the SC gap. We show that the magnitude of the gap on both the hole and electron pockets follows the symmetry of the $d_{y z}$ orbital weight, while FS segments with predominantly $d_{x z}$ or $d_{x y}$ orbital character do not show observable gaps. We then present the theoretical solution to the linearized SC gap equation for a tight-binding model that quantitatively reproduces not only the band dispersions observed in ARPES in the nematic phase but also the orbital characters. In addition, we consider a scenario where the second electron pocket, which is expected to exist but is not observed spectroscopically $[16,18]$, is incoherent and does not contribute to the SC pairing. In this case the anisotropy of the superconducting gap directly scales with the $d_{y z}$ orbital weight on both pockets, in excellent agreement with our experimental results. Our direct observation of a simple relationship between $d_{y z}$ orbital weight and the SC spectral gap can be considered as a strong indication of the efficacy of pairing by spin fluctuations in an $\mathrm{Fe}$ based SC, while the result also highlights the critical impact of an incoherent electron pocket in the nematic phase of FeSe.

Single crystals were grown by chemical vapor transport [11]. ARPES measurements were performed at the I05 beamline at Diamond Light Source [19]. The base temperature reached was $3.7 \mathrm{~K}$ at the sample position. In the best case, we achieve a total instrumental resolution of approximately $2 \mathrm{meV}$ at $37 \mathrm{eV}$ photon energy, with a more typical resolution of $3 \mathrm{meV}$. 

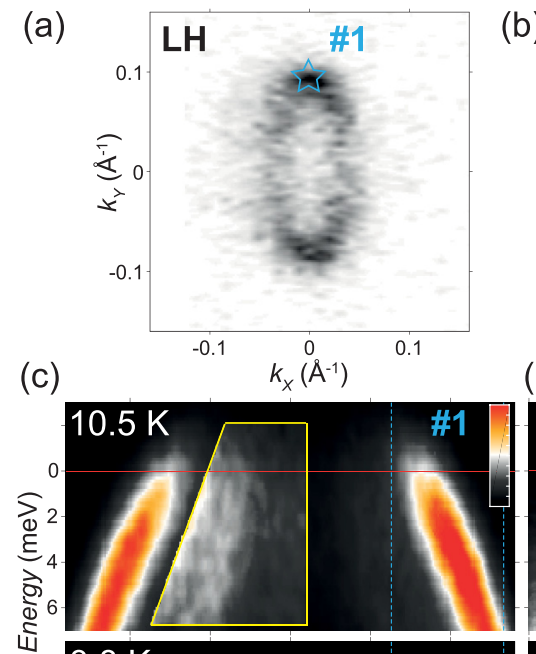

(b)
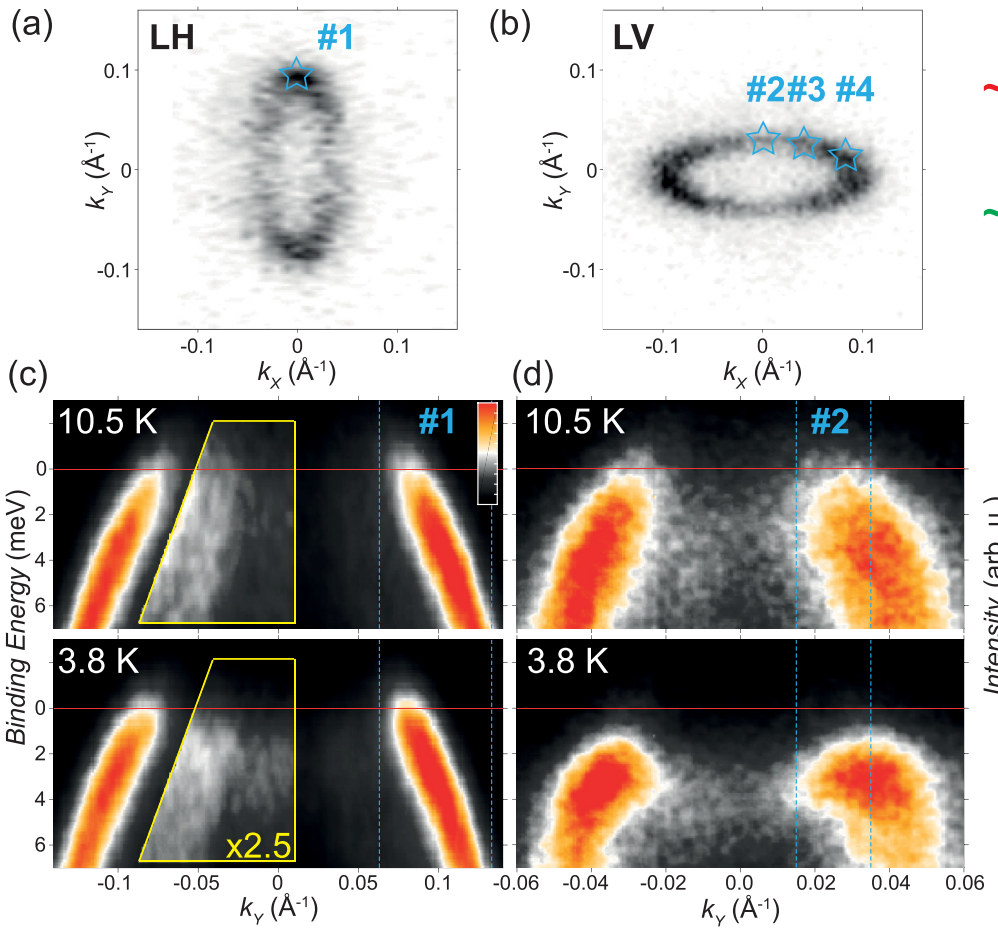

(f)

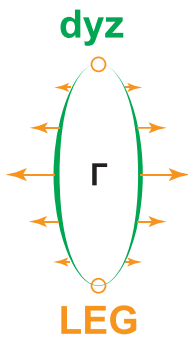

(h)

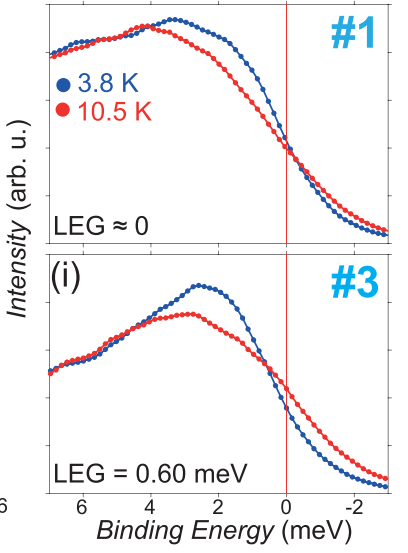

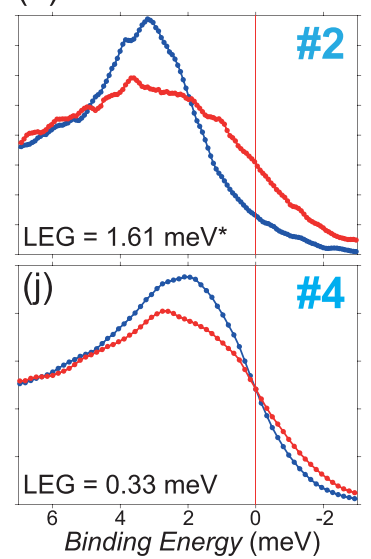

FIG. 1. (a), (b) FS maps of twinned FeSe samples around the $\Gamma$ point $(37 \mathrm{eV})$, in both linear polarizations. (c) High-symmetry dispersions above and below $T_{c}$. (d) High-symmetry dispersion in LV, highlighting the shorter axis of the ellipse. (e) Schematic of the distribution of orbital weights. (f) Schematic correlation between the LEG and the $d_{y z}$ orbital character. (g), (h) EDCs integrated in small regions [cyan dashed lines in (c) and (d)] around $k_{F}$. (i), (j) EDCs at positions shown in (b), off the high-symmetry axes. Note that (d) and (h) are obtained with a higher resolution than other plots.

In Figs. 1(a) and 1(b), we present FS maps at the $\Gamma$ point. The sample is not strained and is twinned, so a superposition of signals from the elliptical hole pocket from two nematic domains is expected. Strikingly, however, one can select which ellipse is primarily observed by switching the polarization. The matrix elements at the zone center are determined by the parity of the orbitals [20]; linear horizontal polarization (LH) couples strongly to $d_{x z}$ orbitals, whereas linear vertical polarization (LV) highlights the $d_{y z}$. The switching behavior implies that the hole pocket must be dominated by $d_{x z}$ character [21]. For the domain with $a$ oriented horizontally (and where the ellipse is elongated along $b$ [18]), this $d_{x z}$ character couples strongly to LH and therefore the vertical ellipse is observed. For the second domain, the reference frame of the orbitals is rotated, and therefore it is mainly the horizontal ellipse which is observed in LV. The dominance of $d_{x z}$ orbital character and the pronounced ellipticity of the pocket are both direct consequences of nematic order [12], since in the tetragonal phase the pocket is circular with fourfold-symmetric $d_{x z}$ and $d_{y z}$ contributions. Although the $d_{x z}$ is dominant, the switching effect is not perfect, for instance, the inner band can also be observed weakly in the LH high-symmetry cuts in Fig. 1(c). A quantitative analysis of the polarization switching effect [22] suggests that the pocket is $\sim 95 \% d_{x z}$ on the major axis and $82 \%-18 \% d_{x z}-d_{y z}$ on the minor axis, summarized in Fig. 1(e).

By comparing ARPES spectra above and below $T_{c}$, we can determine the SC gap $\Delta_{\mathbf{k}}$ around the Fermi surface. On bands where $\Delta_{\mathbf{k}}$ is finite, one should observe a shift of the "leading edge" to higher binding energies compared with the
Fermi-Dirac cutoff in the normal state due to the lack of single-particle excitations within the gap. Additionally, one will observe a coherence peak at an energy scale close to $\Delta_{\mathbf{k}}$. Here, we use the leading-edge gap (LEG) as a well-defined criterion of the superconducting gap; in the regime where the gap size is comparable to or less than the experimental resolution, the LEG is more reliable than the position of the coherence peak, though in general the LEG will underestimate the "true" gap due to the finite resolution [23].

The high-symmetry cuts in Figs. 1(c) and 1(d) reveal that the SC gap on this elliptical hole pocket is extremely anisotropic. The outer band dispersion in Fig. 1(c) does not show any significant difference through $T_{c}$, and the energy distribution curves (EDCs) integrated in a narrow range around $k_{F}$ in Fig. 1(g) simply reveal a slightly sharper Fermi cutoff at low temperatures; within our resolution, the band is not gapped, though we cannot exclude a very small gap $(\lesssim 0.3 \mathrm{meV})$. By contrast, the inner band, seen weakly in LH in Fig. 1(c) but which dominates in LV in Fig. 1(d), is observed to bend back from the Fermi level in the SC state, forming a Bogoliubov band, which can be traced all the way across the pocket to the opposite $k_{F}$. The observation of fused Bogoliubov dispersions is possible only in a regime where $\Delta$ is comparable to $E_{F}$ [24], as is the case for this particular hole band $\left(E_{F} \sim 10 \mathrm{meV}\right)$. A coherence peak at $3.2 \mathrm{meV}$ is observed, with a LEG of $1.6 \mathrm{meV}$; given the resolution of the measurement, the "true gap" at $3.7 \mathrm{~K}$ is estimated to be $2.1 \mathrm{meV}$ [see Supplemental Material (SM) [22]].

This clear anisotropy, taken together with the reduced LEG seen in the data in Figs. 1(i) and 1(j), indicates that the LEG is 


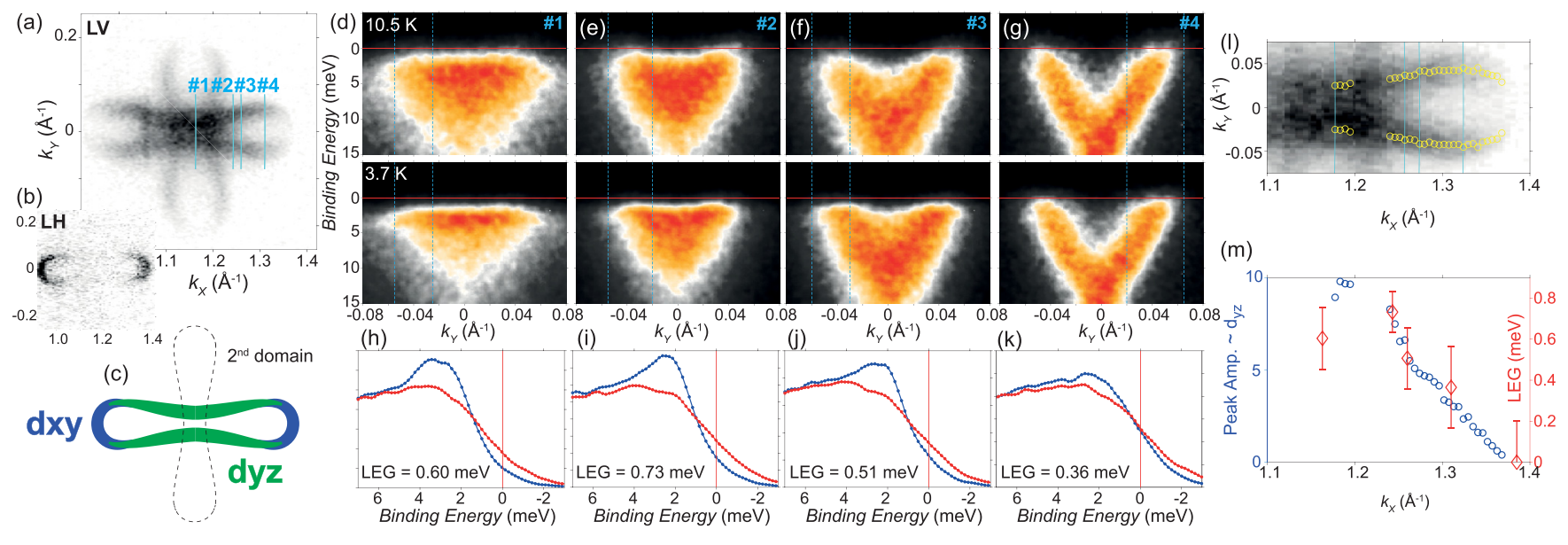

FIG. 2. (a), (b) FS maps of twinned FeSe at the $A$ point $(28 \mathrm{eV})$, schematically represented in (c). (d)-(g) Cuts along $k_{Y}$, above and below $T_{c}$. Analysis of the EDCs in (h)-(k) reveals a SC gap that tends to decrease further away from the center of the peanut. (l) Section of the FS used for the peak-fitting analysis; yellow circles indicate the peak positions. (m) Correlation of $d_{y z}$ peak amplitude from fitting and LEG.

maximal on the minor axis on the ellipse, but shrinks towards zero around the major axis. Thus the gap evolves monotonically around the Fermi surface, and in particular the gap tracks the orbital weight of $d_{y z}$ character, shown schematically in Fig. 1(f). In Fig. 1 we only show data at the $\Gamma$ point, but qualitatively the angular dependence of the gap is similar at the $Z$ point, except for a reduced magnitude (see SM). Thus, even though the $d_{y z}$ orbital weight is in the minority on the hole pocket, it dictates the symmetry of the gap. This is highly unusual: Given the dominance of $d_{x z}$ weight, one might expect a much more uniform gap derived from the pairing of $d_{x z}$ states, but experimentally we see a highly anisotropic gap which is in antiphase with the $d_{x z}$ weight but correlates with the minority $d_{y z}$ weight. This is because there is a separate reservoir of $d_{y z}$ states to pair with on the electron pockethinting at the importance of interband pairing.

In Figs. 2(a)-2(c) we present the experimental and schematic electron pockets, here measured at the $A$ point where the pockets are largest. The understanding of ARPES spectra of the electron pockets has been highly disputed [13,18,25-27]. As discussed in the SM, there is a disagreement between experimentalists on whether a single orthorhombic domain has two electron pockets, as would be expected from calculations $[13,26]$, or one electron pocket. The latter scenario was found in high-resolution ARPES measurements on detwinned samples, which showed, unexpectedly, that in one domain only the peanut-shaped electron pocket oriented along the $a$ axis is detected [18]. Our interpretation is that the FS map in Fig. 2(a) observes just two peanuts, one from each nematic domain in the twinned sample. The full implication of this one electron pocket structure will be discussed later, but we note in the schematic diagram in Fig. 2(c) that the observed peanut has largely $d_{y z}$ orbital character which couples to LV in this geometry, while the tips of the peanut have $d_{x y}$ character, which can be detected in $\mathrm{LH}$ polarization.

The electron pocket not only has a complex shape but also has a strongly anisotropic gap. The high-symmetry cut across the $A$ point in Figs. 2(d) and 2(h) shows a LEG of $0.60 \mathrm{meV}$, a factor of 1.5-2 smaller than the maximum LEG at $\Gamma$. Moving away from the high-symmetry point, in Figs. 2(e) and 2(i) the LEG initially becomes slightly larger but beyond that the general trend is for the gap to reduce as a function of $k_{X}$. Cut No. 4 [Figs. 2(g) and 2(k)] corresponds to where the band is largest, and also where the Fermi velocity is maximal. Here, the gap becomes very small. At the tips of the pocket with $d_{x y}$ character we do not observe any gap. We have additionally confirmed that the center of the pocket is gapped with a similar magnitude at the $M$ point (see SM).

Crucially, the reduction in the size of the gap correlates with a reduced intensity in the FS map, as a function of $k_{X}$ away from the $A$ point. In Fig. 2(m) we present the peak amplitude as a function of $k_{X}$, derived from fitting the FS map in Fig. 2(1). Assuming a constant matrix element, this quantity is proportional to the $d_{y z}$ orbital character. Thus in this geometry we have a rather direct probe of $d_{y z}$ orbital weight, which is found to decrease continuously along the length of the peanut. We can therefore directly show that the magnitude of the LEG, superposed on Fig. 2(m), correlates very well with the $d_{y z}$ orbital weight [28].

We now focus on solving the linearized gap equation in FeSe for spin-fluctuation mediated pairing $[5,6,29,30]$. In this approach it is important to not only accurately model the dispersion of the states present near the Fermi level, but also the orbital character of those states. Our tight-binding model is based on the experimental electronic structure of FeSe in the tetragonal phase [31] (see SM). In order to adapt this for the nematic state of FeSe, we require three key components: (1) a nematic order parameter which correctly describes the symmetry and energy separations of the bands at low temperatures, (2) the inclusion of spin-orbit coupling which mixes the $d_{x z}$ and $d_{y z}$ orbital characters at the hole pocket, and, most critically, (3) the inclusion of only the one experimentally observed electron pocket in the pairing calculations.

In Fig. 3(a) we plot the model Fermi surface in the $k_{z}=0$ plane. These pockets correctly describe the shape, size, and curvature of the low-temperature FS observed by ARPES [11-13,18], with a smoothly elliptical hole pocket and a peanut-shaped electron pocket; for details of the nematic order parameter, see SM. In Fig. 3(b) we present the corresponding 


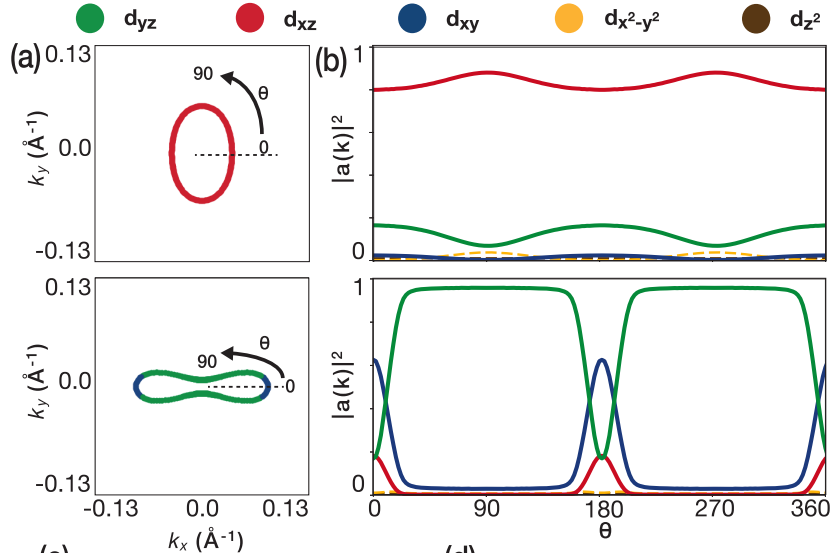

(c) $\quad k_{x}\left(\AA^{-1}\right)$

(d) One electron pocket
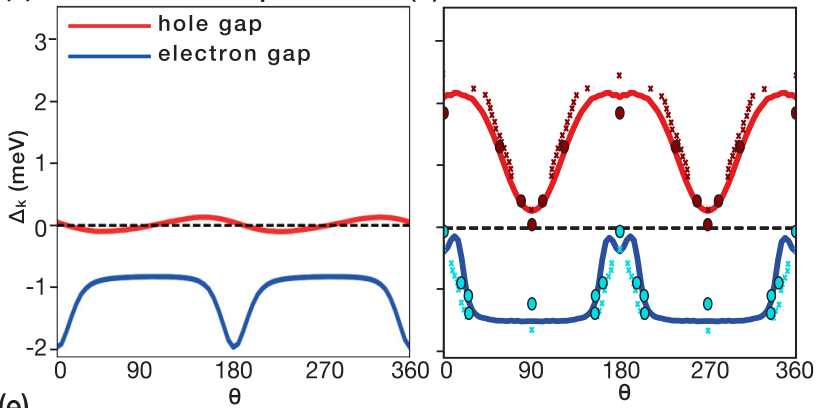

(e)

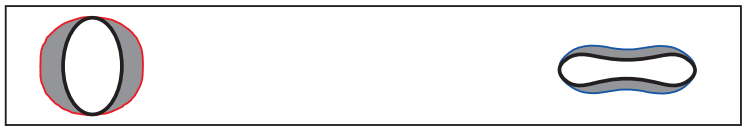

FIG. 3. (a) Closeup of the hole pocket (top) and electron pocket (bottom) at $k_{z}=0$. The color code describes the maximum orbital character and the axes are defined relative to the center of the pockets with $(0,0)$ for the hole pocket and $(\pi, 0)$ for the electron pocket. (b) Orbital characters as a function of angle for the hole pocket (top) and electron pocket (bottom). (c) Momentum dependence of the gap structure for one hole pocket and two electron pockets with $U=0.3 \mathrm{eV}$. (d) The same as (c) but repeated without the electron pocket located at $(0, \pi)$. The colored circles correspond to the ARPES data while the crosses are taken from quasiparticle interference experiments [16]. (e) Representation of the results of (d) around the FS.

orbital characters. For the hole pocket, the orbital character is predominantly of $d_{x z}$ weight, however, there is a contribution of the $d_{y z}$ weight which reaches $16.5 \%$ of the total weight along the minor axis, in agreement with the experimental determination from Fig. 1(e). For the electron pocket at $(\pi, 0)$, the dominant orbital character is $d_{y z}$ with the ends of the peanut being mostly $d_{x y}$.

It can be seen in Fig. 3(c) that when all states are considered in the linearized gap equation (see SM), including the expected electron pocket at $(0, \pi)$, the gap structure does not reproduce the experimental results. The gap on the hole pocket is predicted to have nodes and be much smaller in magnitude than the gap at the $(\pi, 0)$ electron pocket, which broadly follows the $d_{x y}$ orbital character. However, when we remove the contribution to SC pairing from the electron pocket at $(0, \pi)$, we obtain a highly anisotropic gap structure very close to the experimental results, as presented in Fig. 3(d). Here, the hole pocket is nodeless but with a strong anisotropy which follows the minority $d_{y z}$ orbital character shown in Fig. 3(b). The gap function at the electron pocket is also nodeless, with a reduced magnitude and opposite sign to the hole pocket, and closely follows the $d_{y z}$ orbital weight [32]. The success of this calculation shows that as long as one carefully accounts for the details of the experimental FS, including spin-orbit coupling (see SM), nematic order, and considering only the one observed electron pocket, this gap structure which follows the $d_{y z}$ weight with a sign change between the pockets is naturally the leading instability, within spin-fluctuation pairing theory. Thus we have provided an alternative explanation for the anisotropy of the gap structure, which does not require any artificial suppression of quasiparticle weights of certain orbitals [14,16,33] (see SM). Instead, the gap is dictated mainly by the topology and orbital character of the FS.

Our results can be considered as an independent verification of the gap structure determined by Bogoliubov quasiparticle interference measurements [16] and specific heat experiments [34,35]. Previously, Xu et al. [36] found the gap anisotropy at the $Z$ point in $\mathrm{FeSe}_{0.93} \mathrm{~S}_{0.07}$, but here we have extended this to measure the gap on the electron pockets. Additionally, two groups have recently reported the SC gap structure on the hole pocket of FeSe using laser ARPES: Liu et al. reported a similar gap anisotropy to us [17], while some details vary in Hashimoto et al. [37]. Gaps on both the hole and electron pockets were also recently reported by Kushnirenko et al. [26], also using synchrotron ARPES. They find similar in-plane anisotropy of the gap to that reported here, though notably we find that the gap on the hole pocket is largest at the $\Gamma$ point (see SM).

In summary, we have measured the full gap structure and have shown both experimentally and theoretically a direct link between the $d_{y z}$ orbital content and the gap magnitude. While the relationship between the $d_{y z}$ orbital and the gap is theoretically complex, the fact that such a link exists provides strong evidence for spin-fluctuation mediated superconductivity in FeSe. More precisely, it is evidence that the pairing interactions derive from the local, instantaneous, and repulsive Coulomb interactions, in sharp contrast to the retarded, attractive, and orbitally agnostic electron-phonon pairing. Finally, our results also emphasize the impact of an incoherent electron pocket in FeSe.

We thank P. D. C. King, M. Hoesch, A. V. Chubukov, R. M. Fernandes, A. I. Coldea, and L. Fanfarillo for useful discussions. We thank Diamond Light Source for access to Beamline I05 (Proposal No. SI17532) that contributed to the results presented here.
[1] D. S. Inosov, J. T. Park, P. Bourges, D. L. Sun, Y. Sidis, A. Schneidewind, K. Hradil, D. Haug, C. T. Lin, B. Keimer, and V. Hinkov, Normal-state spin dynamics and temperature-dependent spin-resonance energy in optimally doped $\mathrm{BaCo}_{x} \mathrm{Fe}_{2} \mathrm{As}_{2}$, Nat. Phys. 6, 178 (2009). 
[2] M. Ma, P. Bourges, Y. Sidis, Y. Xu, S. Li, B. Hu, J. Li, F. Wang, and Y. Li, Prominent Role of Spin-Orbit Coupling in FeSe Revealed by Inelastic Neutron Scattering, Phys. Rev. X 7, 021025 (2017).

[3] K. Hashimoto, S. Kasahara, R. Katsumata, Y. Mizukami, M. Yamashita, H. Ikeda, T. Terashima, A. Carrington, Y. Matsuda, and T. Shibauchi, Nodal Versus Nodeless Behaviors of the Order Parameters of LiFeP and LiFeAs Superconductors from Magnetic Penetration-Depth Measurements, Phys. Rev. Lett. 108, 047003 (2012).

[4] P. J. Hirschfeld, D. Altenfeld, I. Eremin, and I. I. Mazin, Robust determination of the superconducting gap sign structure via quasiparticle interference, Phys. Rev. B 92, 184513 (2015).

[5] P. J. Hirschfeld, M. M. Korshunov, and I. I. Mazin, Gap symmetry and structure of Fe-based superconductors, Rep. Prog. Phys. 74, 124508 (2011).

[6] S. Graser, T. A. Maier, P. J. Hirschfeld, and D. J. Scalapino, Near-degeneracy of several pairing channels in multiorbital models for the Fe pnictides, New J. Phys. 11, 025016 (2009).

[7] A. M. Oles, Antiferromagnetism and correlation of electrons in transition metals, Phys. Rev. B 28, 327 (1983).

[8] T. Takimoto, T. Hotta, and K. Ueda, Strong-coupling theory of superconductivity in a degenerate Hubbard model, Phys. Rev. B 69, 104504 (2004).

[9] A. V. Chubukov, Pairing mechanism in Fe-based superconductors, Annu. Rev. Condens. Matter Phys. 3, 57 (2012).

[10] A. E. Böhmer and A. Kreisel, Nematicity, magnetism and superconductivity in FeSe, J. Phys.: Condens. Matter 30, 023001 (2018)

[11] M. D. Watson, T. K. Kim, A. A. Haghighirad, N. R. Davies, A. McCollam, A. Narayanan, S. F. Blake, Y. L. Chen, S. Ghannadzadeh, A. J. Schofield, M. Hoesch, C. Meingast, T. Wolf, and A. I. Coldea, Emergence of the nematic electronic state in FeSe, Phys. Rev. B 91, 155106 (2015).

[12] M. D. Watson, T. K. Kim, L. C. Rhodes, M. Eschrig, M. Hoesch, A. A. Haghighirad, and A. I. Coldea, Evidence for unidirectional nematic bond ordering in FeSe, Phys. Rev. B 94, 201107 (2016).

[13] A. Fedorov, A. Yaresko, T. K. Kim, Y. Kushnirenko, E. Haubold, T. Wolf, M. Hoesch, A. Grüneis, B. Büchner, and S. V. Borisenko, Effect of nematic ordering on electronic structure of FeSe, Sci. Rep. 6, 36834 (2016).

[14] A. Kreisel, B. M. Andersen, P. O. Sprau, A. Kostin, J. C. Séamus Davis, and P. J. Hirschfeld, Orbital selective pairing and gap structures of iron-based superconductors, Phys. Rev. B 95, 174504 (2017).

[15] J. Kang, R. M. Fernandes, and A. Chubukov, Superconductivity in FeSe: The Role of Nematic Order, Phys. Rev. Lett. 120, 267001 (2018).

[16] P. O. Sprau, A. Kostin, A. Kreisel, A. E. Böhmer, V. Taufour, P. C. Canfield, S. Mukherjee, P. J. Hirschfeld, B. M. Andersen, and J. C. Séamus Davis, Discovery of orbital-selective Cooper pairing in FeSe, Science 357, 75 (2017).

[17] D. Liu, C. Li, J. Huang, B. Lei, L. Wang, X. Wu, B. Shen, Q. Gao, Y. Zhang, X. Liu, Y. Hu, Y. Xu, A. Liang, J. Liu, P. Ai, L. Zhao, S. He, L. Yu, G. Liu, Y. Mao, X. Dong, X. Jia, F. Zhang, S. Zhang, F. Yang, Z. Wang, Q. Peng, Y. Shi, J. Hu, T. Xiang, X. Chen, Z. Xu, C. Chen, and X. J. Zhou, Orbital Origin of Extremely Anisotropic Superconducting Gap in Nematic Phase of FeSe Superconductor, Phys. Rev. X 8, 031033 (2018).
[18] M. D. Watson, A. A. Haghighirad, L. C. Rhodes, M. Hoesch, and T. K. Kim, Electronic anisotropies revealed by detwinned angle-resolved photo-emission spectroscopy measurements of FeSe, New J. Phys. 19, 103021 (2017).

[19] M. Hoesch, T. K. Kim, P. Dudin, H. Wang, S. Scott, P. Harris, S. Patel, M. Matthews, D. Hawkins, S. G. Alcock, T. Richter, J. J. Mudd, M. Basham, L. Pratt, P. Leicester, E. C. Longhi, A. Tamai, and F. Baumberger, A facility for the analysis of the electronic structures of solids and their surfaces by synchrotron radiation photoelectron spectroscopy, Rev. Sci. Instrum. 88, 013106 (2017).

[20] V. Brouet, M. F. Jensen, P. H. Lin, A. Taleb-Ibrahimi, P. Le Fèvre, F. Bertran, C. H. Lin, W. Ku, A. Forget, and D. Colson, Impact of the two $\mathrm{Fe}$ unit cell on the electronic structure measured by ARPES in iron pnictides, Phys. Rev. B 86, 075123 (2012).

[21] In the nematic phase, we specify $x$ and $y$ to be along the $a$ and $b(a>b)$ axes, respectively.

[22] See Supplemental Material at http://link.aps.org/supplemental/ 10.1103/PhysRevB.98.180503 for methodology.

[23] D. V. Evtushinsky, D. S. Inosov, V. B. Zabolotnyy, A. Koitzsch, M. Knupfer, B. Büchner, M. S. Viazovska, G. L. Sun, V. Hinkov, A. V. Boris, C. T. Lin, B. Keimer, A. Varykhalov, A. A. Kordyuk, and S. V. Borisenko, Momentum dependence of the superconducting gap in $\mathrm{Ba}_{1-x} \mathrm{~K}_{x} \mathrm{Fe}_{2} \mathrm{As}_{2}$, Phys. Rev. B 79, 054517 (2009).

[24] D. V. Evtushinsky, T. K. Kim, A. A. Kordyuk, V. B. Zabolotnyy, B. Büchner, A. V. Boris, D. L. Sun, C. T. Lin, H. Q. Luo, Z. S. Wang, H. H. Wen, R. Follath, and S. V. Borisenko, Fusion of bogoliubons in $\mathrm{Ba}_{1-x} \mathrm{~K}_{x} \mathrm{Fe}_{2} \mathrm{As}_{2}$ and similarity of energy scales in high temperature superconductors, arXiv:1106.4584.

[25] A. I. Coldea and M. D. Watson, The key ingredients of the electronic structure of FeSe, Annu. Rev. Condens. Matter Phys. 9, 125 (2018).

[26] Y. S. Kushnirenko, A. V. Fedorov, E. Haubold, S. Thirupathaiah, T. Wolf, S. Aswartham, I. Morozov, T. K. Kim, B. Büchner, and S. V. Borisenko, Three-dimensional superconducting gap in FeSe from angle-resolved photoemission spectroscopy, Phys. Rev. B 97, 180501 (2018).

[27] Y. Suzuki, T. Shimojima, T. Sonobe, A. Nakamura, M. Sakano, H. Tsuji, J. Omachi, K. Yoshioka, M. Kuwata-Gonokami, T. Watashige, R. Kobayashi, S. Kasahara, T. Shibauchi, Y. Matsuda, Y. Yamakawa, H. Kontani, and K. Ishizaka, Momentum-dependent sign inversion of orbital order in superconducting FeSe, Phys. Rev. B 92, 205117 (2015).

[28] It is worth noting that, even in the case where the pairing interaction is momentum and orbital independent, some gap anisotropy would be induced due to density-of-states factors associated with the complex and anisotropic Fermi surfaces; one would typically expect a larger gap in regions with smaller density of states, i.e., larger Fermi velocity. However, on the electron pockets we find that the gap is smallest on the sections with the highest Fermi velocity. This further substantiates our conclusion that the gap primarily follows the orbital character of the bands, with the details of the band dispersions only playing a secondary role.

[29] D. J. Scalapino, A common thread: The pairing interaction for unconventional superconductors, Rev. Mod. Phys. 84, 1383 (2012). 
[30] T. Saito, Y. Yamakawa, S. Onari, and H. Kontani, Revisit of the orbital-fluctuation-mediated superconductivity in LiFeAs: Nontrivial spin-orbit interaction effects on the band structure and superconducting gap function, Phys. Rev. B 92, 134522 (2015).

[31] L. C. Rhodes, M. D. Watson, A. A. Haghighirad, M. Eschrig, and T. K. Kim, Strongly enhanced temperature dependence of the chemical potential in FeSe, Phys. Rev. B 95, 195111 (2017).

[32] In these results, the normalized eigenvector $g(\mathbf{k})$ has been scaled by a single constant to best fit the experimental gap values as observed in this work and by Bogoliubov quasiparticle interference experiments.

[33] A. Kostin, P. O. Sprau, A. Kreisel, Y. X. Chong, A. E. Böhmer, P. C. Canfield, P. J. Hirschfeld, B. M. Andersen, and J. C. S. Davis, Imaging orbital-selective quasiparticles in the Hund's metal state of FeSe, Nat. Mater. 17, 869 (2018).

[34] Y. Sun, S. Kittaka, S. Nakamura, T. Sakakibara, K. Irie, T. Nomoto, K. Machida, J. Chen, and T. Tamegai, Gap structure of
FeSe determined by angle-resolved specific heat measurements in applied rotating magnetic field, Phys. Rev. B 96, 220505 (2017).

[35] F. Hardy, M. He, L. Wang, T. Wolf, P. Schweiss, M. Merz, M. Barth, P. Adelmann, R. Eder, A. A. Haghighirad, and C. Meingast, Nodal gaps in the nematic superconductor FeSe from heat capacity, arXiv:1807.07907.

[36] H. C. Xu, X. H. Niu, D. F. Xu, J. Jiang, Q. Yao, Q. Y. Chen, Q. Song, M. Abdel-Hafiez, D. A. Chareev, A. N. Vasiliev, Q. S. Wang, H. L. Wo, J. Zhao, R. Peng, and D. L. Feng, Highly Anisotropic and Twofold Symmetric Superconducting Gap in Nematically Ordered $\mathrm{FeSe}_{0.93} \mathrm{~S}_{0.07}$, Phys. Rev. Lett. 117, 157003 (2016).

[37] T. Hashimoto, Y. Ota, H. Q. Yamamoto, Y. Suzuki, T. Shimojima, S. Watanabe, C. Chen, S. Kasahara, Y. Matsuda, T. Shibauchi, K. Okazaki, and S. Shin, Superconducting gap anisotropy sensitive to nematic domains in FeSe, Nat. Commun. 9, 282 (2018). 